\title{
Teores de Fenóis Totais e Taninos nas Cascas de Angico-vermelho (Anadenanthera peregrina)
}

\author{
Caroline Junqueira Sartori ${ }^{1}$, Ana Hortência Fonsêca Castro ${ }^{2}$, Fabio Akira Mori ${ }^{3}$ \\ ${ }^{1}$ Programa de Pós-graduação em Ciência e Tecnologia da Madeira, Departamento de Ciências Florestais, \\ Universidade Federal de Lavras - UFLA, Lavras/MG, Brasil \\ ${ }^{2}$ Departamento de Farmácia, Universidade Federal de São João Del Rey - UFSJ, Divinópolis/MG, Brasil \\ ${ }^{3}$ Departamento de Ciências Florestais, Universidade Federal de Lavras - UFLA, Lavras/MG, Brasil
}

\section{RESUMO}

Este trabalho teve como objetivo quantificar fenóis totais e taninos nas cascas de angicovermelho (Anadenanthera peregrina) em diferentes classes de diâmetro, além de comparar duas metodologias de quantificação de fenóis. Foram coletadas cascas de 35 árvores, distribuídas em sete classes diamétricas. As cascas foram secas ao ar livre, trituradas e peneiradas, sendo utilizado o material retido na peneira de 60 mesh. Para a extração dos fenóis, utilizou-se metanol:água (50\%). O doseamento de fenóis totais foi realizado pelos métodos de Folin-Denis e Folin-Ciocalteau modificados, e para taninos, pelo método difusão radial. Não foi encontrada diferença estatística entre as metodologias de quantificação de fenóis. Verificou-se aumento nos teores de fenóis e taninos com o aumento do diâmetro das árvores.

Palavras-chave: cascas, fenóis, taninos.

\section{Content of Total Phenols and Tannins in Barks of Anadenanthera peregrina}

\begin{abstract}
This study aimed to quantify total phenols and tannins in barks of Anadenanthera peregrina in different diameter classes and compare two methods of quantification of phenols. Barks of thirty-five trees, distributed in seven diameter classes, were collected. They were air-dried, ground and sieved; the material retained on 60 mesh sieve was used. Methanol:water (50\%) solution was used for the extraction of phenols. Quantification of total phenols was performed by the modified Folin-Denis and Folin-Ciocalteau methods, while tannins were quantified by the radial diffusion method. No statistically significant differences were found between the phenol quantification methodologies. Increased contents of phenols and tannins were verified as the diameter of trees increased.
\end{abstract}

Keywords: barks, phenols, tannins. 
$179-8087.061113$

0980 (impresso $)^{1 . ~ I N T R O D U C ̧ A ̃ O ~}$ 79-8087 (online)

Comumente conhecida como angico ou angicovermelho, a Anadenanthera peregrina é uma espécie pioneira, pertencente à família Fabaceae, subfamília Mimosoideae, que possui ampla distribuição geográfica. Ocorre em florestas semidecíduas e na transição com o Cerrado, nos Estados de Tocantins, Goiás, Bahia, Minas Gerais, Rio de Janeiro, São Paulo, Mato Grosso e Mato Grosso do Sul (Lorenzi, 1998).

Diversas são as espécies de angico ocorrentes no Brasil, das quais se extrai, das cascas, os taninos (Afonso, 2008). Segundo o mesmo autor, a casca de angico é um dos principais produtos não madeireiros ocorrentes no Cerrado, sendo fonte natural de compostos fenólicos. A utilização das cascas de angico-vermelho promove um aproveitamento de resíduos e agrega valor, tendo em vista a ampla gama de utilização dos compostos fenólicos.

Os compostos fenólicos são originados do metabolismo secundário das plantas, sendo essenciais para o seu crescimento e a reprodução; além disto, formam-se em condições de estresse, como infecções, ferimentos e radiações ultravioleta (Naczk \& Shahidi, 2004). A diversidade estrutural destes compostos deve-se à grande variedade de combinações que ocorre na natureza e os compostos resultantes são chamados de polifenóis (Harborne, 1989). Segundo Taiz \& Zeiger (2004), os fenóis vegetais constituem um grupo quimicamente heterogêneo, com aproximadamente dez mil compostos. Dentre estes, destacam-se os flavonoides, ácidos fenólicos, fenóis simples, cumarinas, taninos e ligninas. Esses compostos fenólicos possuem estrutura variável e, com isso, são multifuncionais (Shahidi \& Naczk, 1995).

Os taninos vegetais ou naturais são, por definição, substâncias que possuem a propriedade de se associar e de se combinar com proteínas e com certos polióis. Esse comportamento é a base das propriedades tanantes, que os taninos exercem sobre o colágeno da pele dos animais ao curso de sua transformação em couro (Pizzi, 1993). Os taninos ocorrem amplamente nos vegetais, porém sua extração comercial é realizada da casca e/ou do cerne da árvore, partes onde são encontrados em maiores teores (Hemingway, 1989). E, para a planta, os taninos são responsáveis pela defesa contra os herbívoros e doenças patogênicas (Harborne et al., 1991).

Os taninos estão presentes na maioria das plantas e podem variar de concentração nos tecidos vegetais, dependendo da idade, do órgão da planta coletado, da época ou, ainda, do local de coleta. São poucos os estudos de quantificação de fenóis e taninos nas cascas em diferentes diâmetros, e tal estudo é de importância, pois verifica a viabilidade de utilização de indivíduos oriundos de desbaste florestal, promovendo um maior valor agregado às cascas.

A extração de compostos fenólicos é simples, em decorrência de sua solubilidade em solventes orgânicos polares e/ou água, sendo a água muito utilizada, por causa da economia e eficiência; porém, o tipo, a concentração de solvente e a temperatura de extração são específicos de cada espécie e órgão da planta.

São várias as metodologias para quantificação de compostos fenólicos em laboratório, destacando-se os ensaios de precipitação de metais ou proteínas, e os ensaios colorimétricos; observe-se que os colorimétricos são os mais comuns.

O objetivo deste trabalho foi quantificar os teores de fenóis totais e taninos nas cascas de angicovermelho (Anadenanthera peregrina) em diferentes diâmetros, bem como verificar a semelhança entre duas metodologias de quantificação de fenóis totais.

\section{MATERIAL E MÉTODOS}

\subsection{Caracterização da área de estudo}

O plantio de angico pertence a uma área experimental do Campus da Universidade Federal de Lavras (UFLA), localizado em Lavras-MG. A cidade de Lavras encontra-se na região sul do Estado de Minas Gerais, à latitude de $21^{\circ} 14^{\prime}$ sul e longitude $45^{\circ} 00^{\prime}$ oeste, a uma altitude média de $900 \mathrm{~m}$. Segundo a classificação de Köppen, o clima é Cwa. A temperatura média anual é de $19,4^{\circ} \mathrm{C}$ e o total anual de precipitação pluvial de $1.530 \mathrm{~mm}$ (Brasil, 1992). 


\subsection{Coleta e preparo do material}

Coletaram-se, em julho de 2011, cascas de angico-vermelho [Anadenanthera peregrina (Benth) Speg.] na região correspondente ao diâmetro à altura do peito (DAP), tomado a 1,30 m do solo. A coleta foi realizada com o auxílio de facão, retirando-se lascas de aproximadamente $10 \mathrm{~cm}$ de comprimento por $3 \mathrm{~cm}$ de largura, de maneira intercalada, ao longo da circunferência do fuste, de modo a evitar o contorno total do mesmo. Foram coletadas amostras de cinco árvores por classe diamétrica, conforme Tabela 1. Os indivíduos coletados não pertenciam à borda do plantio.

As cascas foram secas ao ar livre, moídas em moinho de martelo e peneiradas em peneiras de $40 \mathrm{e}$ 60 mesh, sendo utilizado o material retido na peneira de 60 mesh. Após a moagem e a classificação das amostras, as mesmas foram armazenadas em sacos de papel abertos e mantidas em sala de climatização com temperatura de $20 \pm 2^{\circ} \mathrm{C}$ e umidade relativa de $60 \pm 5 \%$, até obterem massa constante, para a determinação da umidade.

\subsection{Preparo dos extratos}

Foi utilizado o correspondente a $200 \mathrm{mg}$ de casca seca e moída, a qual foi extraída em $10 \mathrm{~mL}$ de metanol:água (50\%), em maceração a frio e agitação constante por um período de 4 horas, segundo metodologia descrita por Castro et al. (2009). O extrato foi filtrado em papel filtro e o volume completado para $10 \mathrm{~mL}$ com metanol:água (50\%).

\subsection{Teor de fenóis totais pelo método de Folin- Denis}

A curva de calibração foi preparada com o emprego de solução padrão de ácido tânico na concentração de $0,1 \mathrm{mg} / 1.000 \mathrm{~mL}$. Para a quantificação de fenóis totais, foram pipetados 0,05 $\mathrm{mL}$ do extrato, 4,2 $\mathrm{mL}$ de água destilada, $0,25 \mathrm{~mL}$ de reagente Folin-Denis e $0,5 \mathrm{~mL}$ de solução saturada de carbonato de sódio, formando uma mistura de cor azul. Após 30 minutos, foi realizada a leitura da absorbância em espectrofotômetro a $760 \mathrm{~nm}$. As quantificações foram feitas em triplicata e o resultado expresso em porcentagem de ácido tânico, por grama de matéria seca.

\subsection{Teor de fenóis totais pelo método de Folin- Ciocalteau}

Utilizou-se metodologia de Waterhouse (2002). Para preparo da curva padrão, foi utilizada solução alcoólica de ácido tânico. Para a quantificação de fenóis nas amostras, foi retirado $0,5 \mathrm{~mL}$ de cada extrato, acrescido de $2,5 \mathrm{~mL}$ da solução de FolinCiocalteau a $10 \%(\mathrm{v} / \mathrm{v})$ e $2,0 \mathrm{~mL}$ da solução de carbonato de sódio a $4 \%(\mathrm{~m} / \mathrm{v})$, tendo sido protegidos da luz; após 30 minutos, foi medida a absorbância em espectrofotômetro, a $760 \mathrm{~nm}$. As determinações foram realizadas em triplicata e o resultado expresso de forma semelhante ao empregado para o método de Folin-Denis.

Tabela 1. Características dendrométricas do plantio de Anadenanthera peregrina e dos indivíduos utilizados na etapa experimental

Table 1. Dendrometric characteristics of the Anadenanthera peregrina plantation and of the individuals used on experimental stage.

\begin{tabular}{cccc}
$\begin{array}{c}\text { Intervalo das classes } \\
\text { diamétricas } \mathbf{( c m})\end{array}$ & $\begin{array}{c}\text { Valor central das classes } \\
\text { diamétricas } \mathbf{( c m})\end{array}$ & $\begin{array}{c}\text { Diâmetro médio das } \\
\text { árvores } \mathbf{( c m})\end{array}$ & $\begin{array}{c}\text { Altura média das árvores } \\
(\mathbf{m})\end{array}$ \\
\hline 5 a 9,99 & 7,5 & $7,3(9,52 \%)$ & $8,0(14,43 \%)$ \\
10 a 14,99 & 12,5 & $11,9(8,22 \%)$ & $10,9(14,65 \%)$ \\
\hline 15 a 19,99 & 17,5 & $16,8(2,47 \%)$ & $14,0(12,44 \%)$ \\
20 a 24,99 & 22,5 & $22,7(4,26 \%)$ & $14,6(7,92 \%)$ \\
\hline 25 a 29,99 & 27,5 & $27,6(5,35 \%)$ & $15,8(13,18 \%)$ \\
\hline 30 a 34,99 & 32,5 & $31,1(3,44 \%)$ & $17,0(12,25 \%)$ \\
\hline 35 a 39,99 & 37,5 & $37,4(2,70 \%)$ & $18,3(5,26 \%)$ \\
\hline
\end{tabular}

Os valores entre parênteses correspondem ao coeficiente de variação de diâmetro e altura entre as cinco árvores coletadas de cada classe diamétrica. 


\subsection{Teor de taninos totais por meio do método de difusão radial}

Utilizou-se o método de difusão radial, segundo Hagerman (1987). Uma solução de $50 \mathrm{mM}$ de ácido acético e $60 \mu \mathrm{M}$ de ácido ascórbico foi preparada, sendo o pH ajustado para 5,0 pela adição de $\mathrm{NaOH}$ (10\%). O gel foi preparado com agarose (tipo I) $1 \%$ $(\mathrm{p} / \mathrm{v})$, na solução descrita previamente. A mistura foi aquecida sob agitação até entrar em ebulição para a completa homogeneização da agarose e resfriada até a temperatura de $45^{\circ} \mathrm{C}$, sendo então adicionada posteriormente albumina sérica bovina (BSA) na concentração de $0,1 \%(\mathrm{p} / \mathrm{v})$. Alíquotas de $15,1 \mathrm{~mL}$ da solução foram distribuídas em Placas de Petri com $13,5 \mathrm{~cm}$ de diâmetro e mantidas em bancadas niveladas para obter camadas uniformes de gel até solidificação total. Com o emprego de um perfurador de 4,5 $\mathrm{mm}$ de diâmetro, foram realizados orifícios, distando $2 \mathrm{~cm}$ um do outro e das bordas das placas.

Para a obtenção da curva padrão, preparouse uma solução aquosa de ácido tânico com concentração de $25 \mathrm{mg} / \mathrm{mL}$. Alíquotas de 1, 4, 8, 12, 16,20 e $24 \mu \mathrm{L}$ foram dispostas nos poços em triplicata e os diâmetros dos halos, medidos após 72 horas.

Para o preparo das amostras, foram retirados $5 \mathrm{~mL}$ dos extratos previamente preparados, os quais foram concentrados em chapa a $50^{\circ} \mathrm{C}$ e posteriormente ressuspendidos com $0,5 \mathrm{~mL}$ de metanol a $50 \%(\mathrm{v} / \mathrm{v})$. Para a dosagem de taninos nas amostras, foram utilizados $15 \mu \mathrm{L}$ de cada extrato, aplicados aos poços em triplicata, e esperadas 72 horas para reação.
O resultado foi expresso de forma semelhante ao empregado para os métodos de Folin-Denis e FolinCiocalteau.

\subsection{Análise estatística}

Foi realizado o teste de Bartlett a 5\%, para verificar a homogeneidade de variâncias, e também o teste de Shapiro-Wilk a 5\%, para verificar a normalidade das variâncias. Na avaliação do experimento, empregou-se um delineamento inteiramente casualizado em esquema fatorial, sendo duas metodologias distintas para quantificação de fenóis totais em sete classes de diâmetro, ao considerar o valor central de cada classe diamétrica. Para o fator quantitativo (centro de classe), foi utilizada a análise de regressão por polinômios ortogonais, em um nível de significância de 5\%. Adotou-se um delineamento inteiramente casualizado para analisar a influência do fator diâmetro nos valores de fenóis totais e taninos.

Foram realizadas correlações lineares de Pearson entre metodologias de fenóis totais e entre cada metodologia e teores de taninos, além de análise de regressão e ajustes dos modelos lineares, e teste $\mathrm{F}$ a $5 \%$ de significância.

\section{RESULTADOS E DISCUSSÃO}

Na Tabela 2, encontram-se os valores médios de fenóis totais obtidos ao se empregarem as metodologias de Folin-Denis e Folin-Ciocalteau,

Tabela 2. Valores de fenóis totais pelos métodos de Folin-Denis e Folin-Ciocalteau, e taninos totais por difusão radial, nas sete classes diamétricas.

Table 2. Values of total phenols by methods of Folin-Denis and Folin-Ciocalteau and tannins by radial difusion, in seven diameter classes.

\begin{tabular}{cccc}
$\begin{array}{c}\text { VCD } \\
(\mathbf{c m})\end{array}$ & $\begin{array}{c}\text { Fenol total } \\
\text { Folin-Denis (\%) }\end{array}$ & $\begin{array}{c}\text { Fenol total } \\
\text { Folin-Ciocalteau (\%) }\end{array}$ & $\begin{array}{c}\text { Taninos Totais Difusão } \\
\text { Radial (\%) }\end{array}$ \\
\hline 7,5 & $10,91(13,14)$ & $9,94(10,26)$ & $7,52(10,01)$ \\
12,5 & $11,43(13,62)$ & $10,83(13,64)$ & $8,12(9,68)$ \\
17,5 & $11,50(9,21)$ & $11,22(9,07)$ & $9,03(7,10)$ \\
22,5 & $12,45(17,20)$ & $11,77(14,48)$ & $9,13(11,11)$ \\
\hline 27,5 & $12,23(8,13)$ & $12,30(7,90)$ & $10,08(15,46)$ \\
32,5 & $13,01(12,17)$ & $12,33(13,95)$ & $11,79(15,58)$ \\
\hline 37,5 & $14,09(7,64)$ & $13,71(7,75)$ & $12,05(10,12)$ \\
\hline Média & 12,23 & 11,73 & 9,67 \\
\hline CV $(\%)$ & 8,85 & 10,37 & 17,93 \\
\hline
\end{tabular}

${ }^{*}$ VCD: Valor central da classe diamétrica. Os valores entre parênteses correspondem aos coeficientes de variação dentro da classe diamétrica. 
bem como valores médios de taninos por classe diamétrica. Os valores entre parênteses correspondem aos coeficientes de variação (\%) dentro das classes de diâmetro.

Apesar de os valores de fenóis totais por FolinDenis terem sido, na maioria das amostras, maiores do que os valores de fenóis por Folin-Ciocalteau, não foi encontrada diferença estatística entre as metodologias de quantificação de fenóis totais, bem como interação entre as metodologias e as classes diamétricas. Encontrou-se diferença apenas para a fonte de variação 'classe diamétrica' tanto para a variável 'fenol total' quanto para taninos.

A produção média de taninos totais por difusão radial foi de 9,67\%. Mariano et al. (2010) encontraram valores médios de teor de taninos condensados de 14,76\% para casca de Anadenanthera peregrina, resultado maior do que o obtido neste estudo pelo método de difusão radial. Tais resultados maiores encontrados pelos autores citados podem ser por causa da metodologia empregada (teste de Stiasny), que leva em consideração, na mensuração de compostos fenólicos, os fenóis não tânicos, superestimando os valores em taninos. Segundo Hagerman (1987), uma grande vantagem da metodologia de difusão radial é que fenóis não tânicos, como flavonoides, ácidos benzoicos ou ácidos hidroxicinâmicos, não interferem no ensaio.

As maiores produções de fenóis totais e taninos foram obtidas na classe correspondente aovalor central de $37,5 \mathrm{~cm}$, e os menores valores, na de $7,5 \mathrm{~cm}$. Na Figura 1, está apresentada a regressão linear simples, as equações de regressão de primeiro grau e seus coeficientes de determinação, entre valores de fenóis totais $\left(\mathrm{R}^{2}=0,952\right)$ e valores de taninos $\left(\mathrm{R}^{2}=0,955\right)$, por valor central da classe, respectivamente. Nota-se um incremento nos teores de fenóis totais e taninos com aumento do diâmetro do fuste das árvores. Nesta relação, é verificada a influência do diâmetro na produção desses compostos.

Cabral et al. (2010) estudaram a influência da altura, do diâmetro e da espessura de casca de aroeira (Myracrodruon urundeuva) e quixabeira (Sideroxylon obtusifolium), nos teores de taninos e fenóis totais, pelos métodos de difusão radial e Folin-Ciocalteau, respectivamente. No estudo, as classes diamétricas foram divididas de 3 a 4,99 cm, de 5 a $6,99 \mathrm{~cm}$, de 7 a $8,99 \mathrm{~cm}$, de 9 a $10,99 \mathrm{~cm}$, e de 11 a $12,99 \mathrm{~cm}$, para a aroeira, e de 0 a $1,99 \mathrm{~cm}$, de 2 a $3,99 \mathrm{~cm}$, e de 4 a $5,99 \mathrm{~cm}$, para a quixabeira, respectivamente. Para ambas as espécies, não foi encontrada nenhuma relação entre os níveis de taninos e fenóis, e os parâmetros dendrométricos avaliados.

Teixeira et al. (1990) avaliaram a variação do teor de taninos da casca do caule de barbatimão (Stryphnodendron adstringens) em função de classes diamétricas. Essa separação foi realizada em grupos de indivíduos com caules de diâmetro de 5 a 9,99 cm, 10 a 14,99 cm, e 15 a 19,99 cm. Nesse estudo, também não foi encontrada evidência da influência do diâmetro à altura do peito nos teores de taninos.

$\mathrm{Na}$ Figura 2, está representada a relação entre as metodologias de quantificação de fenóis totais,

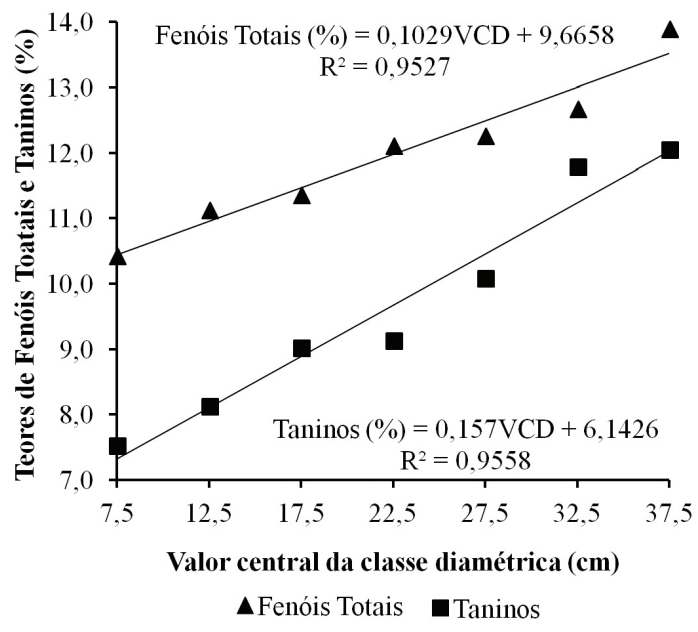

Figura 1. Relação entre fenóis totais e taninos com valor central da classe diamétrica.

Figure 1. Relationship between total phenols and tannin with central value of the diametric class.

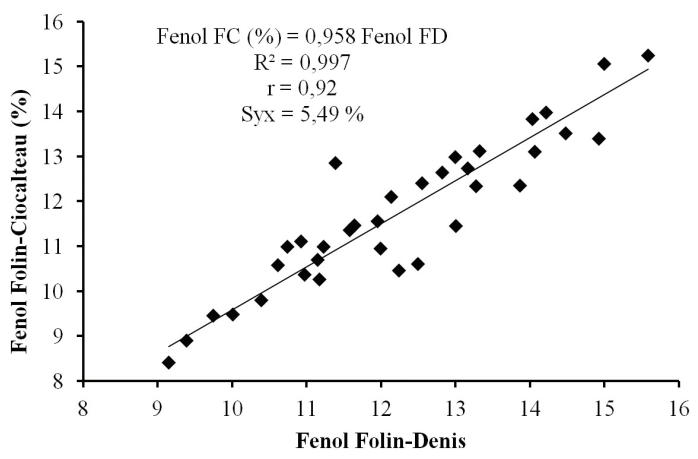

Figura 2. Relação entre as metodologias de quantificação de fenóis totais.

Figura 2. Relationship between methodologies of total phenols quantification. 
por Folin-Denis e Folin-Ciocalteau, e a equação de regressão. Foram encontrados elevados coeficientes de determinação e correlação de Pearson $\left(\mathrm{R}^{2}=0,997\right.$; $r=0,92)$. A relação de $99,7 \%$ confirma a semelhança encontrada entre as metodologias de quantificação de fenóis.

Verifica-se que os valores de fenóis por FolinDenissão, em sua maioria, maiores do que os valores de fenóis por Folin-Ciocalteau. Este fato provavelmente pode ser devido ao fato de o método de Folin-Denis não fazer distinção entre compostos fenólicos e outros materiais redutores ou antioxidantes, como o ácido ascórbico, formando precipitados que interferem na leitura espectrofotométrica (Schofield et al., 2001); observa-se, porém, que as metodologias forneceram resultados semelhantes estatisticamente.

\section{CONCLUSÕES}

As metodologias de quantificação de fenóis são estatisticamente iguais. Indica-se a metodologia de Folin-Denis, uma vez que a mesma utiliza uma menor quantidade de reagentes, o que resulta em menor custo e menor geração de resíduos químicos.

As maiores produções de fenóis e taninos foram encontradas nas classes de maiores diâmetros, o que torna mais apreciável a extração destes compostos nestas classes.

O método de difusão radial pode ser utilizado com segurança, já que este foi eficaz, pois os valores encontrados para taninos foram menores do que os de fenóis totais. Ressalte-se que este método não necessita de equipamentos sofisticados e é de fácil execução.

\section{STATUS DA SUBMISSÃO}

Recebido: 7 out., 2013

Aceito: 4 maio, 2014

Publicado: 28 ago., 2014

\section{AUTOR(ES) PARA CORRESPONDÊNCIA}

Caroline Junqueira Sartori

Programa de Pós-graduação em Ciência e Tecnologia da Madeira, Departamento de Ciências Florestais, Universidade Federal de Lavras - UFLA, CEP 37200-000, Lavras, MG, Brasil

e-mail: krolsartori@hotmail.com

\section{REFERENNCIAS}

Afonso SR. Análise sócio-econômica da produção de nãomadeireiros no Cerrado brasileiro e o caso da cooperativa de pequi em Japonvar, MG. [dissertação]. Brasília: Departamento de Engenharia Florestal, Universidade de Brasília; 2008.

Brasil. Ministério da Agricultura e Reforma Agrária. Secretaria Nacional de Irrigação. Departamento Nacional de Meteorologia. Normais climatológicas: 1961 1990. Brasília; 1992. 84 p.

Cabral DLV, Peixoto Sobrinho TJS, Amorim ELC, Albuquerque UP. Relationship of biometric parameters on the concentration of tannins in two medicinal plants: a case study. Boletín Latinoamericano y del Caribe de Plantas Medicinales y Aromáticas 2010; 9(5): 368-376.

Castro AHF, Paiva R, Alvarenga AA, Vitor SMM. Calogênese e teores de fenóis e taninos totais em barbatimão [Stryphnodendron adstringens (Mart.) coville]. Ciência e Agrotecnologia 2009; 33(2): 385-390. http://dx.doi.org/10.1590/S1413-70542009000200004

Harborne JB. General procedures and measurement of total phenolics. In: Dey PM, Harborne JB, editors. Methods in plant biochemistry: plant phenolics. London: Academic; 1989. http://dx.doi.org/10.1016/B978-0-12461011-8.50007-X

Harborne JB, Palo RT, Robbins CT. Plant defenses against mammalian herbivore. London: CRC; 1991.

Hagerman AE. Radial diffusion method for determining tannin in plant extracts. Journal of Chemical Ecology 1987; 13(3): 437-448. PMid:24301886. http:// dx.doi.org/10.1007/BF01880091

Hemingway RW. Chemistry and significance of condensed tannins. New York: Plenum; 1989. PMCid:PMC2429847. http://dx.doi.org/10.1007/978-14684-7511-1

Lorenzi H. Árvores brasileiras: manual de identificação e cultivo de plantas arbóreas do Brasil. 2. ed. Nova Odessa: Instituto Plantarum; 1998.

Mariano RF, Mori FA, Goulart SL, Mori CLSO, Dias LMS. Determinação do teor de taninos a partir da casca de angico, Anadenanthera Spp. In: Anais do XII Encontro Brasileiro em Madeiras e em Estruturas de Madeira [CD-ROM]; 2010; Lavras.

Naczk M, Shahidi F. Extraction and analysis of phenolics in food. Journal of Chromatography A 2004; 1(2): 95111. http://dx.doi.org/10.1016/j.chroma.2004.08.059

Pizzi A. Tanin-based adhesives. In: Pizzi A, editor. Wood adhesives: chemistry and techonology. New York: Marcel Dekker; 1993. PMCid:PMC281255

Schofield P, Mbugua DM, Pell AN. Analysis of condensed tannins: a review. Animal Feed Science 
and Technology 2001; 91(1-2): 21-40. http://dx.doi. org/10.1016/S0377-8401(01)00228-0

Shahidi F, Naczk M. Food phenolics: sources, chemistry, effects and applications. Lancaster: Technomic; 1995.

Taiz L, Zeiger E. Fisiologia vegetal. 3. ed. Porto Alegre: Artmed; 2004.
Teixeira ML, Soares AR, Scolforo JRS. Variação do teor de tanino da casca de barbatimão [Stryphnodendron adstringens (Mart.) Coville] em 10 locais de Minas Gerais. Ciência e Prática 1990; 14(2): 229-232.

Waterhouse AL. Polyphenolics: determination of total phenolics. In: Wrolstad RE, editor. Currente protocols in food analytical chemistry. New York: John Wiley; 2002. 